\title{
INTERCONEXIÓN ENTRE REDES ORGANIZACIONALES, ALIANZAS ESTRATÉGICAS Y NEGOCIACIONES Un estudio multicaso
}

\author{
Enrique Louffat Olivares, Ph.D. \\ PROFESOR DE ADMINISTRACIÓN DE ESAN \\ elouffat@esan.edu.pe
}

\section{Resumen}

El presente artículo pretende realizar un análisis centrado en el diseño y dinámica organizacional de las relaciones de interdependencia entre dos o más organizaciones. Con esta intención se establecen dos dimensiones de análisis: los condicionantes, constituidos por los elementos: estrategia, ambiente, tecnología y cultura, y los componentes constituidos por los elementos: departamentalización, actividades de línea/asesoría, descripción de actividades, cadena jerárquica, nivel de centralización/descentralización, amplitud administrativa, comunicación y capacidad decisoria. Luego, para tener una visión actual de la aplicación de este análisis en la práctica organizacional, se realiza una investigación exploratoria de tipo cualitativa en tres grupos corporativos con perfiles de redes/alianzas de tipo horizontal y vertical. Los resultados llevan a comprobar una tendencia positiva en la aceptación y aplicación de esa nueva propuesta en red, lo que implica una profunda evolución de las características del diseño y dinámica organizacional.

\section{Contextualización del fenómeno de estudio}

El inicio del siglo XXI nos permite observar una serie de cambios en el campo organizacional, en su identidad estratégica, estructural y operacional, los cuales han modificado profundamente las bases de las ciencias administrativas, poniendo en jaque sus paradigmas tradicionales. Entre los diversos tipos de cambios organizacio- nales, el relacionado con el diseño organizacional merece ser analizado con mayor detalle ante las nuevas exigencias y necesidades de las relaciones internas y externas. En este sentido, Nadler y Tushman (2000: 69) comentan que los cambios en el ambiente de los negocios desafían las premisas del diseño organizacional. Históricamente, el objetivo de las estructuras organizacionales era institucionalizar la estabilidad; en la empresa del futuro, el 
objetivo de las estructuras organizacionales será institucionalizar el cambio. En ese contexto, ¿cuál sería la estructura organizacional más adecuada, la que permitiría a las organizaciones modernas enfrentar la nueva era de la administración?

Como respuesta a esta interrogante, la literatura viene presentando con gran frecuencia e intensidad la propuesta de una estructura organizacional en red. Así, Savage (1996: 89) comenta que la estructura organizacional del siglo XXI podría tomar la forma de una red. En opinión de Lipnack y Stamps (1992), la red está emergiendo como forma principal de organización en la era de la información, tal como la burocracia selló la era industrial, la jerarquía controló la era de la agricultura y el pequeño grupo dominó en la era nómada. De las afirmaciones de Borys y Jeninson (1989, citado por Kataoka, 1995: 4), Silva (1994: 94-96), y Kanter y Eccles (citado por Silva, 1994: 94-96), se deduce que las formas organizacionales del tipo red son importantes tanto de la perspectiva profesional como de la académica, porque si bien representan nuevas opciones y retos para la gestión, también desafían la capacidad de la teoría existente para describir y explicar sus causas y operaciones. Un aspecto relevante del contexto de actuación de la estructura organizacional en red que merece ser comentado es que, en cuanto al diseño de la estructura tradicional, se limita al estudio y análisis de una única organización, mientras en la estructura organizacional en red es ampliado para el estudio y análisis de dos o más organizaciones en relación simultánea, interactiva y de interdependencia (Peci, 1998: 71; Wildeman, 1999: 77). Es importante también resaltar que las relaciones entre dos o más organizaciones que dan origen a la red pueden establecerse entre proveedo- res, clientes y hasta competidores (Bateman y Snell, 1998: 266).

Dadas las características de las estructuras organizacionales en red, se deduce que, por naturaleza, habría una estrecha correlación entre ellas y las alianzas estratégicas; podría decirse que entre ambas se crea una simbiosis. Para Freire (1998: 465), al preservarse la autonomía de cada organización en la red, esta estructura se asemeja a un entretejido de alianzas estratégicas coordinadas por un órgano central cuya única misión es optimizar el desempeño conjunto. En el mismo sentido, Kanter (2000, citado por Cândido, 2001: 38) afirma que las opciones y exigencias en el actual mundo organizacional produce un escenario donde habrá mayor número de alianzas, sociedades y colaboración extrema en la forma de redes cada vez más flexibles.

Aceptada la compatibilidad entre las estructuras organizacionales en red y las alianzas estratégicas, debemos tener presente que ésta resulta de una negociación permanente que permite planear, organizar, dirigir y controlar el diseño, funcionamiento y evaluación de la estructura organizacional en el contexto de la alianza estratégica. Así, la importancia de la negociación queda supeditada a los cambios en los diseños organizacionales, anotación resaltada por Martinelli y Almeida (1997: 133) cuando se refieren a que la gran fase de reestructuración por la cual vienen pasando las empresas desde la década pasada -con muchas fusiones, adquisiciones, franquicias, emprendimientos conjuntos, conduciendo muchas veces nuevos tipos de arreglos empresariales- hace que la negociación se perciba no en un sentido de conflicto o rivalidad, sino como cooperación, complemento o unión. 


\section{La investigación}

Establecidos estos fundamentos y ante el nuevo contexto de los negocios, se puede observar que las estructuras organizacionales en red, las alianzas estratégicas y las negociaciones son conceptos fuertemente vinculados entre sí pero que precisan ser más explorados y analizados en sus interconexiones y características. En la actualidad puede afirmarse que ellos son conceptos under construction. Por lo tanto, la presente investigación tiene un objetivo general y un conjunto de objetivos específicos:

\section{Objetivo general}

Determinar cuáles son las principales interconexiones y características de los condicionantes y de los componentes de la estructura organizacional en red y sus negociaciones en el contexto de alianzas estratégicas.

\section{Objetivos específicos}

a) Presentar una interconexión conceptual entre estructura organizacional en red, la alianza estratégica y la negociación. b) Analizar la interconexión de los condicionantes: estrategia, ambiente, tecnología y cultura, y su influencia en el surgimiento de la estructura organizacional en red, de las alianzas estratégicas y de sus negociaciones.

c) Analizar las características de los componentes: departamentalización, actividades de línea/asesoría, descripción de actividades, cadena jerárquica, nivel de centralización/descentralización, amplitud administrativa, comunicación, y capacidad decisoria en el diseño de la estructura organizacional en red, teniendo como contexto las alianzas estratégicas desde la perspectiva de la negociación que fue realizada para su diseño e implementación.

\subsection{Bases teóricas}

\section{a) Conceptos}

Ante la diversidad de propuestas sobre terminologías y características del objeto de estudio y teniendo como base tratadistas reconocidos (ver cuadro 1), optamos por construir conceptos propios e interconectados, los cuales han servido de soporte en esta investigación de las tres líneas temáticas propuestas.

\section{Cuadro 1}

\section{Soporte teórico para construcción de conceptos}

\begin{tabular}{|l|l|}
\hline Líneas temáticas & \multicolumn{1}{c|}{ Autores } \\
\hline Estructura organizacional en red & $\begin{array}{l}\text { Amoroso, 1994; Bakers, citado por Silva, 1994; Gerstein, en } \\
\text { Nadler et. al., 1994; Kataoka, 1995; McGuill y Slocum Jr., 1995; } \\
\text { Barnatt, 1996; Louffat, 1999; Wildeman, 1999; Castells, 2000; } \\
\text { Ghoshal y Bartlett, 2000. }\end{array}$ \\
\hline Alianza estratégica & $\begin{array}{l}\text { Amoroso, 1994; Ross y Lorange, 1996; Bateman y Snell, 1998; } \\
\text { Devlin y Bleacky, 1998. }\end{array}$ \\
\hline Negociación & $\begin{array}{l}\text { Sparks, 1992; Pinto, 1994; Cohen y Junqueira, 1986, citado por } \\
\text { Martinelli y Almeida, 1997; Ury, 1999. }\end{array}$ \\
\hline
\end{tabular}


Proponemos a continuación los conceptos que son la base de las definiciones y constructos operacionales de esta investigación.

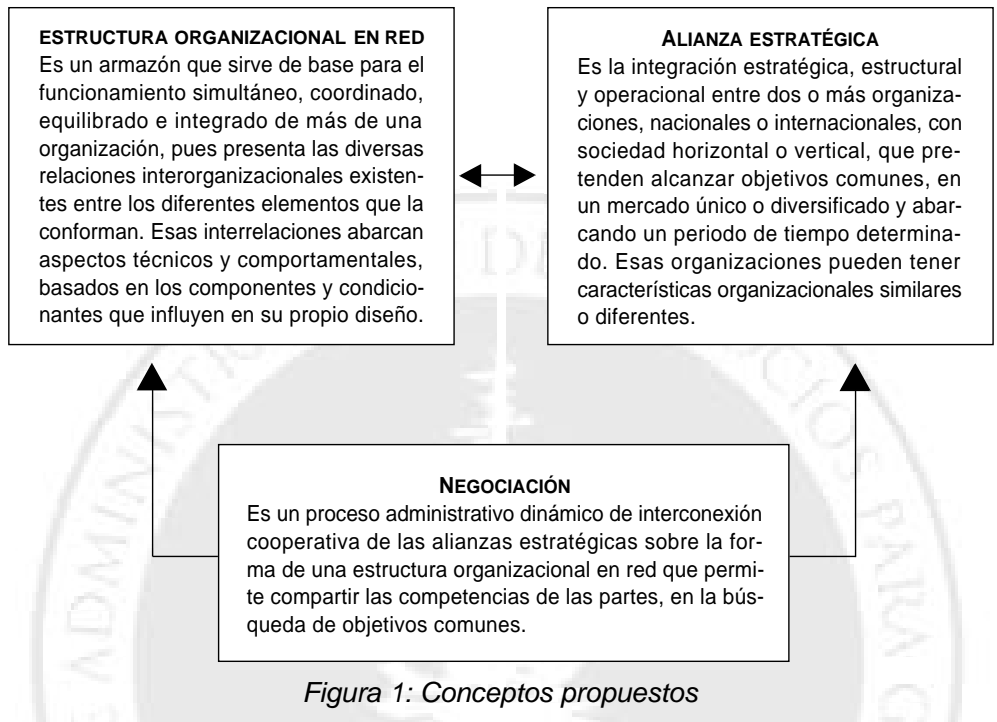

\section{b) Tipología}

En la cuestión de la tipología, también se pueden encontrar diversas propuestas clasificatorias tanto de redes como de alianzas. Con la intención de clarificar y sentar las bases de las relaciones ínterorganizacionales y buscar la uniformidad y conexión terminológica y operacional, se optó por proponer dos tipos básicos de redes/alianza: la horizontal y la vertical.

i) Red/alianza horizontal. Está constituida por organizaciones competidoras que ofrecen el mismo servicio, operación o producto final, desarrollando relaciones de co-opetition (Brandenburger y Nalebuff, 1996). Esta rela- ción paradójica de cooperación versus competición permite que, por un lado, organizaciones rivales acuerden estrategias, estructuras y operaciones específicas y, de otro lado, preserven su independencia e identidad propia.

ii) Red/alianza vertical. Está constituida por organizaciones que actúan de forma complementaria en una cadena de valor, ofreciendo una parte del servicio, operación o producto final. En éstas un conjunto de proveedores y de distribuidores/clientes dirigen sus operaciones para atender a una organización central (productora/organizadora de la red), la cual coordina las acciones de las diversas organizaciones que forman parte de la cadena. 
Otro aspecto digno de comentar es que la tipología propuesta en este estudio, sea horizontal y/o vertical, también adopta una característica complementaria, la cual denominamos de «convergencia operacional» y que presenta el grado de integración/conexión efectiva entre las socias de la alianza/red. Así, se puede observar tres grados básicos de integración (figura 2). El grado 1 se caracteriza por la inauguración de una empresa/sede nueva o por la adaptación de alguna empresa ya existente de alguna de las socias; el grado 2 se caracteriza por el establecimiento de enlaces de tipo ad hoc entre unidades organizacionales, creadas por cada socia para desarrollar operaciones de enlace entre ellas; el grado 3 privilegia la integración independiente total, es decir, las unidades organizacionales rutinarias de cada socia pueden interrelacionarse al instante en los diversos procesos, actividades, niveles.

Es bueno resaltar que en este proceso de gradación de la alianza/red no es im- prescindible seguir un modelo de secuencia lineal. Puede suceder perfectamente, por ejemplo, que una red sea desde el comienzo de su formación del grado 3 sin pasar previamente por los grados 1 y 2 . Conviene resaltar también que no se hace un juicio de valor sobre cuál es el mejor grado de la red, aunque es preciso anotar que el grado ideal de integración operacional es el grado 3.

\subsection{Modelo conceptual}

El modelo conceptual que se usa en la presente investigación está constituido por los siguientes elementos:

- Tres unidades temáticas: estructura organizacional en red, alianza estratégica y negociación.

- Dos categorías de análisis: condicionantes y componentes.

- Doce variables operacionales: estrategia, ambiente, tecnología, cultura, de-

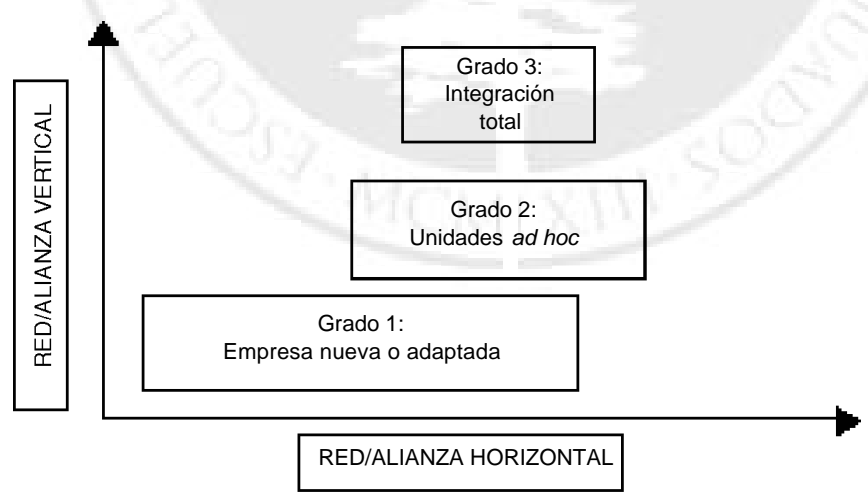

Figura 2: Tipos de redes/alianzas 


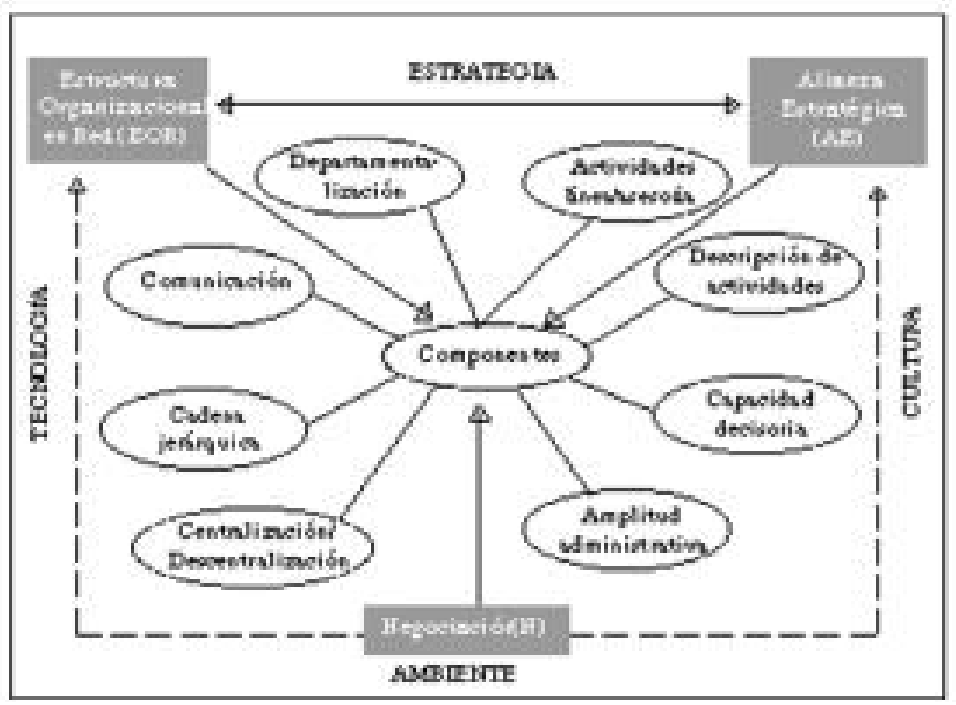

Figura 3: Modelo teórico conceptual

partamentalización, actividades de línea/asesoría, descripción de actividades, cadena jerárquica, nivel de centralización/descentralización, amplitud administrativa, comunicación y capacidad decisoria.

\subsubsection{Los condicionantes}

Los condicionantes son los elementos de origen exógeno, del nivel macroorganizacional, que influyen fuertemente en el surgimiento de una estructura organizacional en red. Son la estrategia, el ambiente, la tecnología y la cultura.

Estrategia.- Este condicionante ejerce influencia sobre la estructura organizacional en red y las alianzas estratégicas en la forma de coaliciones de estructuras basadas en la interdependencia, para alcanzar los objetivos colectivos de la red y los propios intereses de cada socia. Esta interconexión sucede por la necesidad de que las organizaciones busquen sinergias concentrando sus actividades en aquellas que mejor realizan, sus core competences (Prahalad y Hamel, 1990, citado por Silva, 1994; Dyer, Kale y Singh, 2001: 37). Para Savage (1996: 247-249), las visiones organizacionales -en un contexto de red- son un compuesto de varios elementos: diálogos estratégicos, planes, metas, filosofías, factores críticos de relaciones y valores basados en el mercado real y su contexto, que inclusive puede abarcar actividades de internacionalización, ante lo cual adopta modelos estratégicos: multinacional, global, internacional o transnacional (Ghoshal y Bartlett, 2000). La 
interconexión que la negociación establece con la estructura organizacional en red y la alianza estratégica se basa en el hecho de que para poder servir de apoyo en esa relación entre socias, debe actuar como facilitadora de acuerdos que satisfagan los objetivos individuales y conjuntos.

Ambiente.- Este condicionante influye sobre la estructura organizacional en red y las alianzas estratégicas por los continuos cambios en el contexto de las organizaciones modernas, que se basan en una relación de interacción más dinámica con los diversos stakeholders (proveedores, clientes, sindicatos, etc.), lo que significa convergencia de grupos de interés en la red (Piore citado por Silva, 1994: 88). Las redes de alianzas se vuelven la base de la competencia no sólo internamente, sino en la área global. La interconexión que la negociación tiene con la estructura organizacional en red y las alianzas estratégicas se basa en el hecho de que, para poder servir de apoyo en esa relación entre socias, se debe considerar la idea de cooperación interorganizacional con proveedores, clientes y hasta competidores.

Tecnología- Este condicionante influye en la estructura organizacional en red y las alianzas estratégicas a través de la tecnología de la información, elemento fundamental que ofrece la infraestructura necesaria para la emergencia de las redes organizacionales (Peci, 1998: 2-6). De otro lado, la tecnología también se convierte en elemento que favorece la constitución de alianzas estratégicas, ya que generalmente es muy difícil que una única entidad (persona u organización) tenga las capacidades y recursos suficientes para proyectos tecnológicos de gran es- cala; ésta es la principal motivación para el surgimiento de alianzas y redes que compartan actividades para el objetivo común de desarrollar conocimientos específicos (Lewis, 1992: 44). La interconexión que la negociación tiene con la estructura organizacional en red y las alianzas estratégicas se basa en el hecho de que pueden existir tecnologías de información y de comunicación virtual que sirvan de apoyo en las relaciones entre las socias.

Cultura.- Este condicionante influye en la estructura organizacional en red y las alianzas estratégicas porque se vuelve indispensable en el surgimiento y desarrollo de las mismas. En ese sentido, si partimos de la premisa de que toda organización tiene su propia cultura, entonces una tarea vital para el éxito del funcionamiento de la red será conciliar las diversas culturas de las socias (Segil, 1998: 13). Cabe también reflexionar sobre procesos de internacionalización empresarial y el aspecto cultural. Así existen tres opciones o posturas filosófico-culturales: la etnocéntrica, la policéntrica y la geocéntrica (Grosse y Kujawa, 1992: 353-358; Keegan y Green, 1999: 10-13). La interconexión que la negociación establece con la estructura organizacional en red y la alianza estratégica se basa en el hecho del apoyo clave para buscar integrar y conciliar la cultura de cada socia a favor de la red, dejando de lado divergencias, preconceptos y creencias inadecuadas.

\subsubsection{Los componentes}

Los componentes son los elementos de origen endógeno, del nivel microorganizacional, que influyen de forma técnica en el diseño y la operacionalización de una estructura organizacional en red. 
Departamentalización.- Este componente de la estructura organizacional en red en el contexto de alianzas estratégicas se caracteriza por adoptar un agrupamiento por procesos de negocios. Así, las estructuras en red luchan por forjar nuevas alianzas tanto con clientes internos como externos, y para satisfacer las necesidades de éstos buscan mejorías continuas de procesos a lo largo de toda la organización (McGuill y Slocum Jr. 1995: 101). La negociación en este componente busca determinar acuerdos en relación con, entre otros, los siguientes asuntos: ¿cuántos procesos principales se establecerán?, ¿cuántos procesos se desarrollarán en conjunto por las socias?, ¿cuántos procesos serán desarrollados independientemente por cada socia?, ¿cuál será la jerarquía de los procesos?

Actividades de línea/asesoría.- Este componente de la estructura organizacional en red en el contexto de alianzas estratégicas busca la plena interacción, donde todos los miembros estén unidos a través de células de procesos de negocios (Baker, en Nohria y Eccles, 1992: 401). Se resalta el uso de células, porque ellas facilitan la integración de unidades orgánicas en forma de equipos flexibles e integrados en la red. La negociación en este componente busca acuerdos con relación a cuestiones como las siguientes: ¿cuáles serán las competencias de las células?, ¿cuál será el grado de interacción e integración del personal de línea y de asesoría dentro de cada célula e intercélulas?, ¿cuál de las organizaciones socias tendrá un papel asesor o de ejecución en cada célula o proceso de negocios?

Descripción de actividades.- Este componente de la estructura organizacional en red en el contexto de la alianza es- tratégica se caracteriza por una mayor flexibilidad y empowerment de las células (Oliveira, Dalcul y Ruas, 1996: 42-43, citado por Dellagnello y Silva, 2000: 12). Otro aspecto por resaltar es la tendencia a medir los resultados de las diversas tareas, procesos, funciones, actividades, lo que de por sí implica establecer indicadores de desempeño (Lewis, 1992: 109). La negociación en este componente busca acuerdos con relación a, entre otros, los siguientes asuntos: ¿qué tipos de células?, ¿cuáles serán las competencias de las células?, cuáles serán los criterios para formar parte de las células?, ¿cuál será la duración de la célula?, ¿cuál será el grado de $\mathrm{em}$ powerment de la célula?, ¿qué resultados se espera de las células?

Cadena jerárquica.- Este componente de la estructura organizacional en red en el contexto de alianza estratégica se basa en una estructura jerárquica plana (horizontal, achatada) con pocos niveles, privilegiando no tanto las funciones sino valorizando los procesos de negocios integrados. Al contrario de entender a la organización como «cajas de organigramas» mutuamente exclusivos ente sí, preconiza las células, sobreponiéndolas unas a otras como diagramas de Venn (Savage, 1996: 255). La negociación en este componente busca acuerdos con relación a asuntos como los siguientes: ¿cuántos niveles celulares o de procesos jerárquicos se establecerán?, ¿cuál será la relación de poder entre las socias?, ¿cómo se establecerá la relación de jerarquía entre los funcionarios de una y otra socia?

Centralización/descentralización.Este componente de la estructura organizacional en red en el contexto de alianzas estratégicas busca una mayor descentralización, basada en la autoridad técnica de 
las competencias de las diversas células. Ellas se deben responsabilizar por la definición de sus metas, propósitos y misión, conjuntamente con sus planes de proyectos en un banco de datos compartido. Ciertamente, existirán decisiones estratégicas más centralizadas y con delegación de autonomía para las decisiones tácticas y operacionales (Gnyawali y Madhavan, 2001: 434-435). La negociación en este componente busca acuerdos con relación a asuntos como los siguientes: ¿decisiones individuales o por equipos?, ¿cuál es el nivel de desconcentración de las decisiones?, ¿qué criterios se adoptarán para tomar decisiones?

Amplitud administrativa.- Este componente de la estructura organizacional en red en el contexto de alianzas estratégicas se caracteriza por ser más dinámica, en el sentido de que la jefatura de cada célula constituida podría ser temporal y simultánea, y un funcionario de una célula podría formar parte de más de un proceso. Es más difícil establecer alguna fórmula que estandarice un número específico de subordinados adscritos a una jefatura en cada red/alianza (Overholt, 2000: 68-72). La negociación en este componente busca acuerdos en relación con, entre otros, los siguientes asuntos: ¿las jefaturas son integradas y únicas entre las socias?, ¿las jefaturas son en forma rotativa o permanente?, ¿cuál es la cantidad de funcionarios adscritos a la jefatura en cada célula?, ¿un mismo funcionario puede ser jefe de varias células simultáneamente?

Comunicación.- La estructura organizacional en red en el contexto de alianzas estratégicas se caracteriza por tener una comunicación integrada a todo nivel, basada en el soporte de las tecnologías de comunicación e información tipo intranet y extranet, lo que le permite actuar al instante (Albertin, 2000: 28; Castells, 2000: 190-191). La negociación en este componente busca acuerdos en relación con, entre otros, los siguientes asuntos: ¿cómo será la comunicación intranet y extranet?, ¿cuáles los canales de comunicación?, ¿cuáles los medios que serán empleados?

Capacidad decisoria.- La estructura organizacional en red en el contexto de alianzas estratégicas se caracteriza por tomas de decisiones más frecuentes, rápidas y complejas, en ambientes de cambios cada vez más vertiginosos en todos los campos y con leyes de mercado más complejas en sus relaciones de competición y cooperación (Dellagnello y Silva, 2000). En la red, la toma de decisiones es con frecuencia realizada conjuntamente con clientes, proveedores y hasta competidores. Las redes posibilitan tomas de decisiones más rápidas, flexibles e integradas. La negociación en este componente busca acuerdos en relación con, entre otros, los siguientes asuntos: ¿cuál es el nivel de flexibilidad e integración de las decisiones entre las socias?, ¿cuál es el proceso de toma de decisiones conjuntas?, ¿cuáles son las áreas de competencias de las decisiones?

\subsection{Metodología}

La investigación realizada puede ser considerada como una combinación de tres dimensiones metodológicas: una investigación de carácter exploratorio (Gil, 1988: 45-48; Selltiz, 1964: 60), con características cualitativas (Creswell, 1994: 5) aplicadas a estudios de casos (Yin, 1989: 23; Ludke y Andre, 1988: 17-21).

En la teoría y en la práctica se nota que las ideas y aplicaciones propuestas 
sobre interconexiones de redes, alianzas y negociación están muy dispersas y no son claras; de ahí la necesidad de explorar estas bases temáticas de forma interconectada. La elección de una investigación cualitativa obedece a la necesidad de una percepción original, basada en la aplicación de nuestro modelo teórico. Para poder desarrollar la investigación, nada mejor que aplicarla a estudios de casos, donde las particularidades de cada red/alianza nos pueden ayudar a alcanzar una comprensión más clara y técnica del modelo.

\section{El estudio multicaso}

\subsection{Las unidades de análisis}

Teniendo en cuenta las características anotadas anteriormente, para el desarrollo de esta investigación se juzgó conveniente optar por el análisis de tres grupos corporativos mexicanos, los cuales son líderes en sus respectivos mercados y realizan, además, actividades de internacionalización.

Para efectos didácticos, la estructura organizacional de cada uno de estos grupos puede ser agrupada en grandes niveles, como se explicará a continuación.

\section{En el Grupo CEMEX (Cementos} Mexicanos S. A. de C. V.) pueden distinguirse los siguientes niveles:

a) El nivel corporativo, constituido por el CEO (chief executive officer) y las vicepresidencias ejecutivas centrales (Desarrollo y Planeamiento Estratégico), las vicepresidencias (Energía,

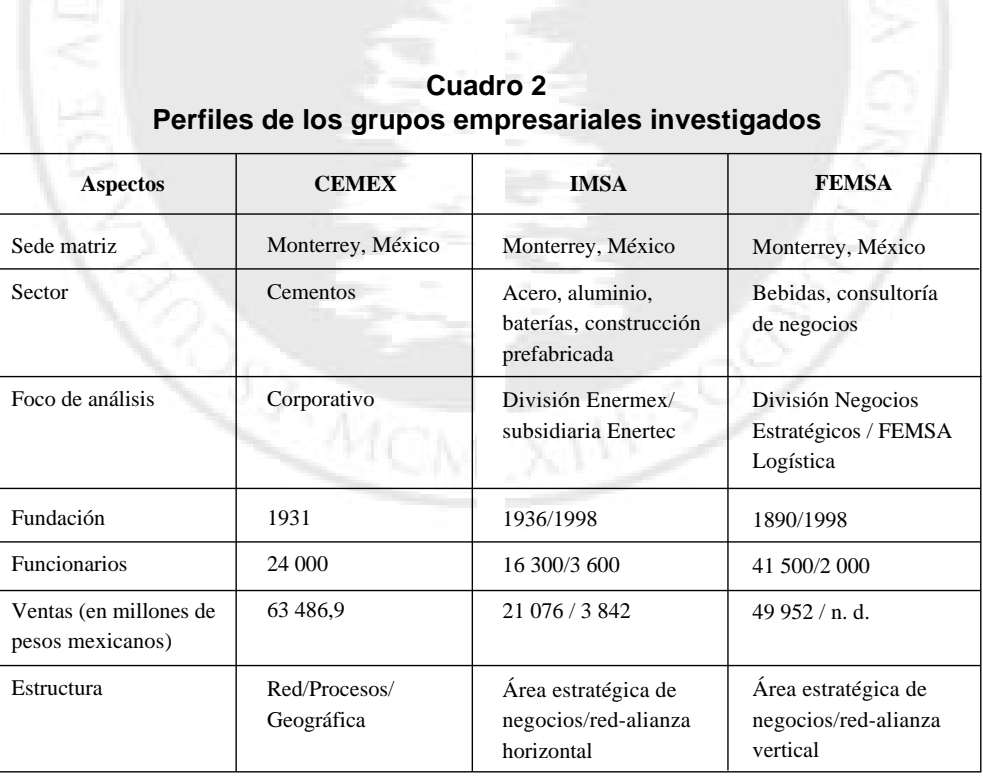


Abastecimiento, CIO, O\&M, Tecnología, Finanzas, Planeamiento Estratégico, Asuntos Legales, Desarrollo de Información, Desarrollo de Negocios de Asia, de África, de América, Comunicación \& Imagen, Administración), las gerencias (Soporte de Administración, Administración de Proyectos) y las direcciones (Administración de Riesgo, Servicios Administrativos, Tributación, Evaluación de Procesos, Seguridad, Servicios Administrativos).

b) El nivel geográfico mundial, constituido por tres grandes presidencias de zonas o áreas mundiales (América del Norte; Europa, África y Asia; América del Sur y El Caribe) y una vicepresidencia de e-business global; presidencias/direcciones en cada país, agrupadas de acuerdo con cada área mundial. Por ejemplo, las presidencias de CEMEX de España, Egipto y Asia están subordinadas a la presidencia de Europa, África y Asia.

c) El nivel País, constituido por la presidencia del respectivo país, la que cuenta con vicepresidencias de apoyo (Planeamiento, Recursos Humanos, Tecnología y Consejería Legal) y vicepresidencias de línea (Operaciones, Comercial, Read Mix, Logística); todas ellas, obviamente, en el país.

En el Grupo IMSA (Industrias Monterrey S. A. de C. V.) pueden observarse los siguientes niveles:

a) El nivel corporativo, constituido por el CEO y las direcciones centrales de apoyo (Jurídica, Finanzas y Recursos Humanos), así como las direcciones de línea en las cuatro áreas estratégi- cas de negocios: Imsa Acero, Enermex, Imsatec e Imsalum.

b) El nivel de las áreas estratégicas de negocios (AEN), de las cuales en este estudio fue escogida específicamente la división Enermex, que con un CEO y sus direcciones de línea (Operaciones, Finanzas, Recursos Humanos, Comercial, Tecnología y Calidad).

c) El nivel de red/alianza estratégica de tipo 1: Enertec, constituida por la red/ alianza entre la división Enermex del grupo IMSA y los grupos Varta (Alemania) y Johnson Controls (Estados Unidos).

En el Grupo FEMSA (Fomento Económico de Monterrey S. A de C. V.) pueden observarse los siguientes niveles:

a) El nivel corporativo, constituido por el CEO, las direcciones centrales de apoyo (Finanzas, Auditoría Interna, Planeamiento de Recursos Humanos) y las direcciones de las tres áreas estratégicas de negocios: FEMSA Cerveza, Coca Cola FEMSA y unidades estratégicas de negocios; y dentro de esta última área, específicamente la empresa FEMSA Logística.

b) El nivel de red/alianza estratégica de tipo vertical, constituida por FEMSA Logística (conformada por la dirección general y las direcciones de línea: Operaciones I, Operaciones II, Operaciones de Almacenes, Comercial y Proyectos, Ingeniería y Compras, Administración, Recursos Humanos y Sistemas) y las organizaciones-socias (clientes) a las cuales ofrece consultoría en gestión de redes de valor. 


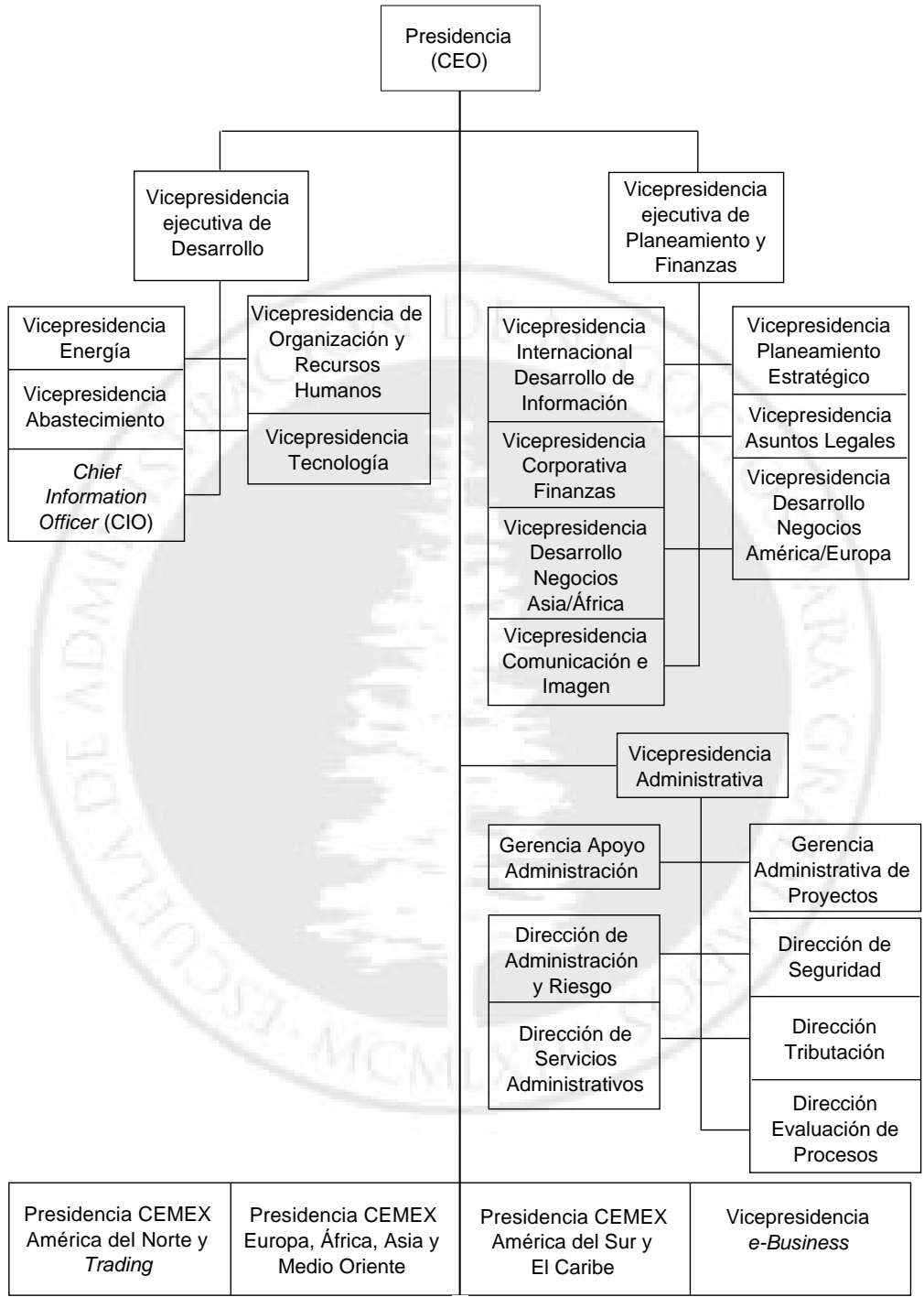

Fuente: Documentos internos del grupo CEMEX.

Figura 4: Organigrama corporativo de CEMEX 


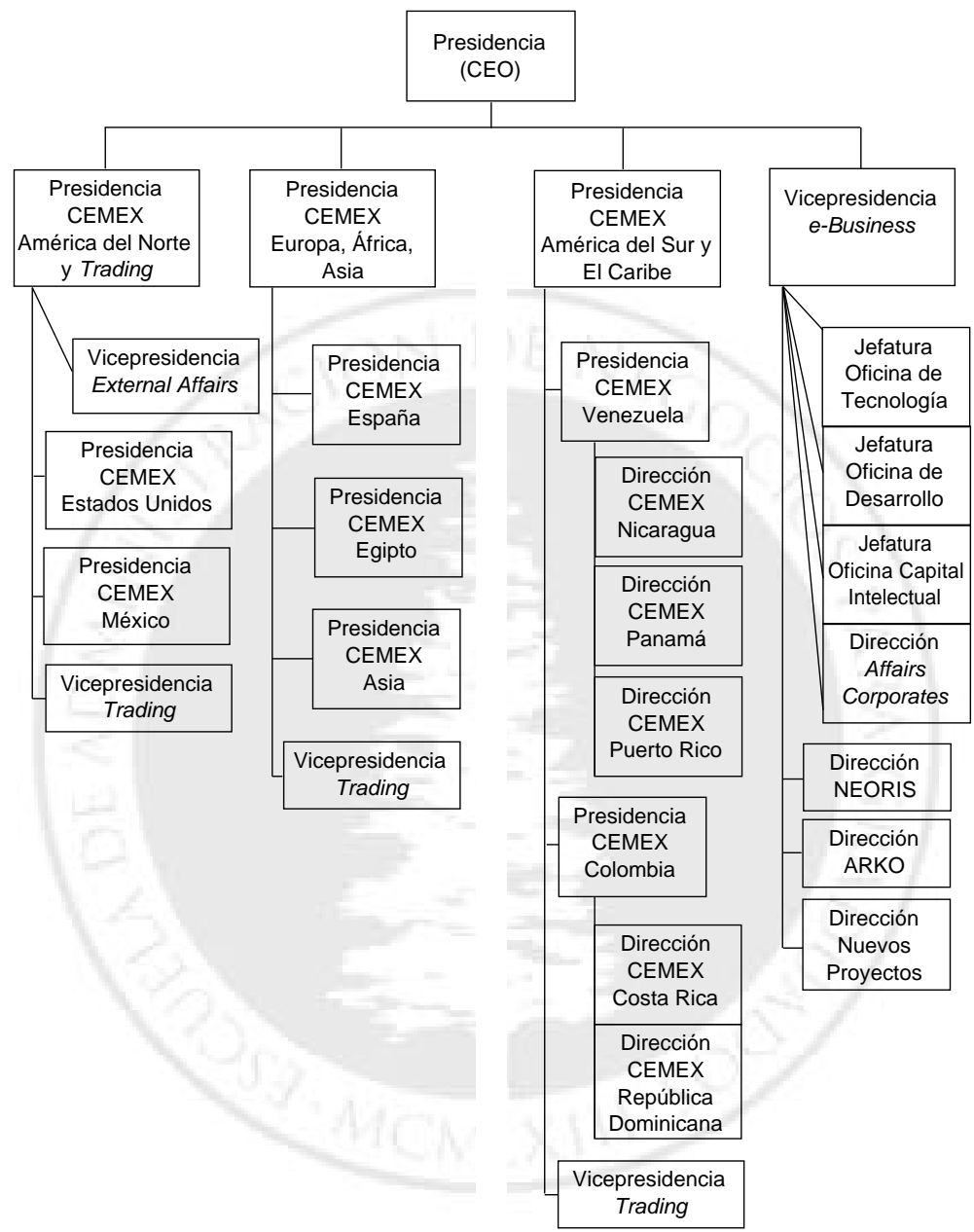

Fuente: Documentos internos del grupo CEMEX.

Figura 5: Organigrama zonas mundiales (geográficas) CEMEX 


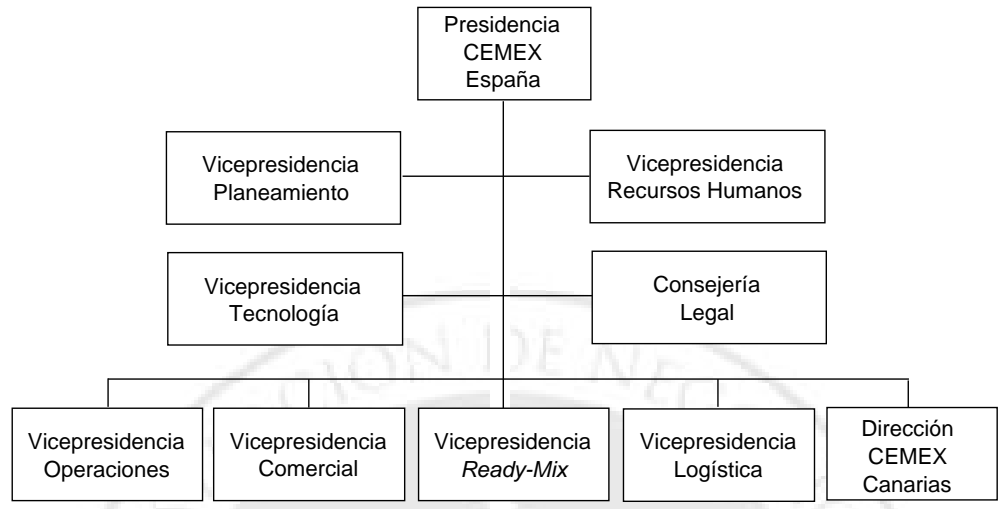

Fuente: Documentos internos del grupo CEMEX.

Figura 6: Organigrama de CEMEX España

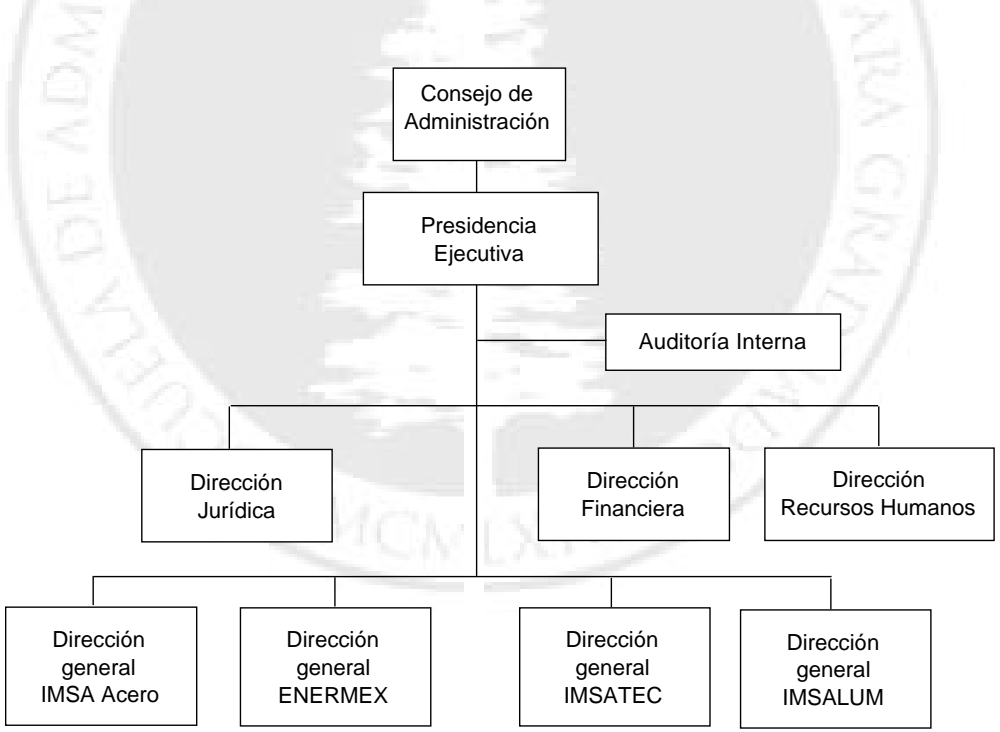

Fuente: Documentos internos del grupo IMSA.

Figura 7: Organigrama corporativo de IMSA 


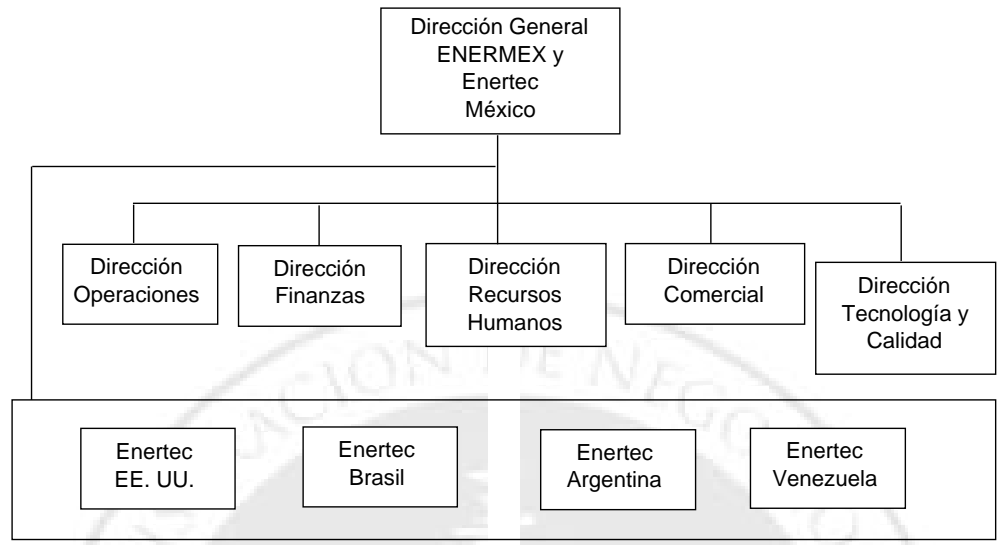

Fuente: Documentos internos del grupo IMSA.

Figura 8: Organigrama de división ENERMEX

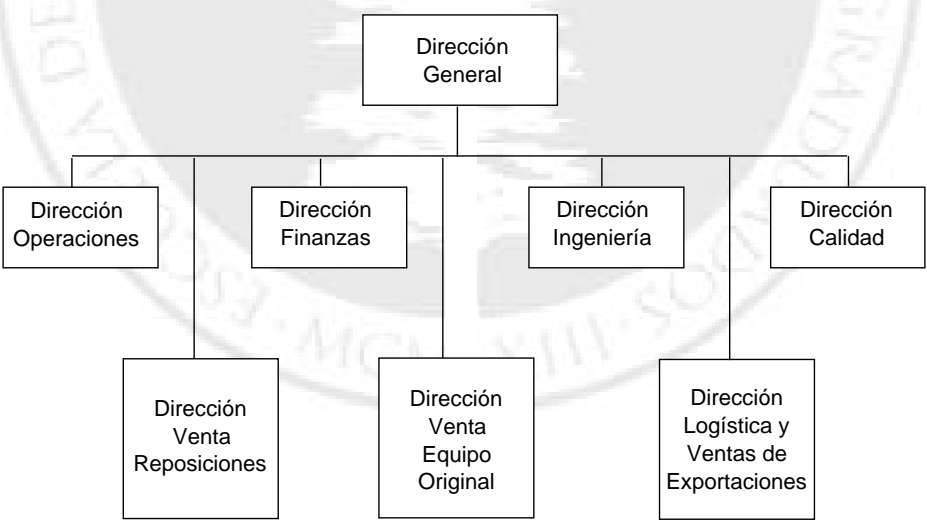

Fuente: Documentos internos del grupo IMSA.

Figura 9: Organigrama Enertec Brasil 


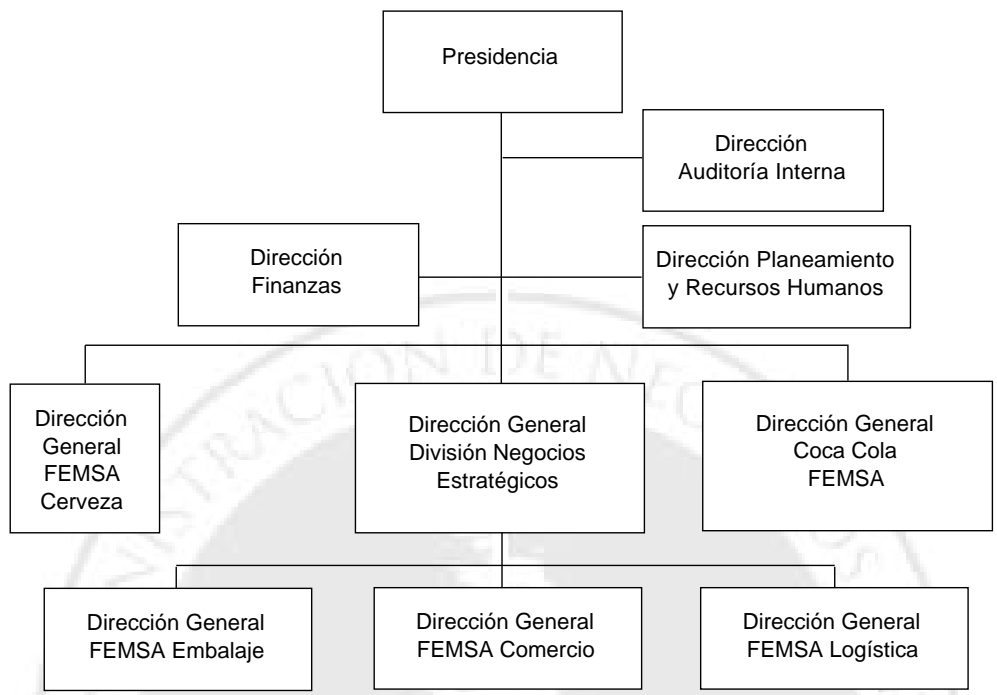

Fuente: Documentos internos del grupo FEMSA.

Figura 10: Organigrama corporativo de FEMSA

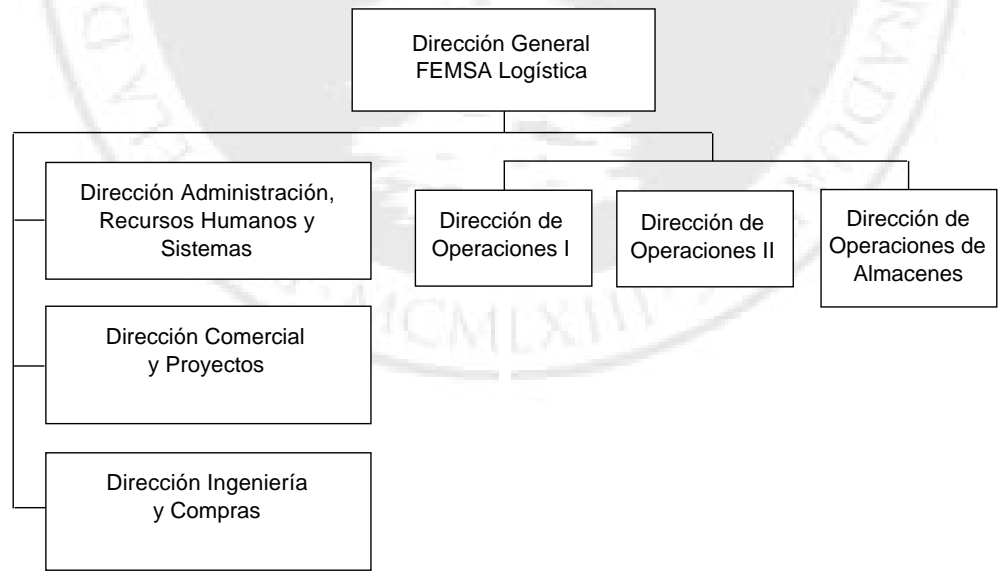

Fuente: Documentos internos de FEMSA logística.

Figura 11: Organigrama de FEMSA Logística 


\subsection{Recolección y tratamiento de los datos}

La recolección de datos se realizó básicamente por medio de entrevistas con trece ejecutivos del nivel de dirección (top) de los tres grupos empresariales (fuentes primarias), así como del análisis de documentos internos y externos a las empresas (fuentes secundarias). Las entrevistas, abiertas semiestructuradas y de una hora y media de duración aproximadamente, fueron aplicadas en la sede de cada organización. Como refuerzo, hubo intercambio de correos electrónicos o llamadas telefónicas para confirmar o complementar alguna información. En cuanto a los documentos analizados, los principales fueron: memorias anuales, organigramas y gráficos de flujos, informes reservados, artículos, entre otros.

Para el análisis de los datos se utilizó la técnica de análisis de contenido. Berelson (citado por Bardin, 1977: 36) la considera como: «una técnica de investigación que, a través de una descripción objetiva, sistemática y cualitativa del contenido manifiesto de las comunicaciones, tiene por finalidad la interpretación de estas comunicaciones». De esta manera se desarrolló el siguiente proceso de análisis:

Etapa 1: Selección de documentos, en la que fueron objeto de análisis las entrevistas transcritas así como los documentos internos y externos.

Etapa 2: Formulación de objetivos/cuestiones, cuya intención era conocer la experiencia de las redes/alianzas de los tres grupos empresariales.

Etapa 3: Categorización, basada en dos dimensiones centrales de análisis, es decir, los condicionantes y los componentes.

Etapa 4: Codificación/unidades de análisis, que consistió en el agrupamiento de las respuestas y/o informaciones obtenidas de acuerdo con las variables o cuestiones referenciales.

Etapa 5: Inferencias/resultados, que consistió en el análisis de tipo inductivo-deductivo, de lo particular a general y viceversa, tratando de consolidar y confirmar la validez y confiabilidad de los hechos encontrados, a través de la confrontación de respuestas e informaciones en cada dimensión y su respectivo componente, en la relación teórico-práctica.

\subsection{Resultados}

Los hallazgos encontrados se presentan en función de cada condicionante y componente.

\subsubsection{Condicionantes}

Estrategia.- Es un condicionante fundamental que influye la configuración en red de los tres grupos. Así, el grupo CEMEX adopta una sólida estrategia de desarrollo global, lo que significa que administra la corporación como una unidad comandada por la matriz, la cual establece una estandarización en sus diversas subsidiarias mundiales. FEMSA Logística no tiene todavía experiencias de internacionalización con operaciones directas; sus actividades procuran un posicionamiento de consolidación local dentro del propio México. En el caso específico de sus redes /alianzas de tipo vertical, sus socios generalmente actúan en sectores complementarios encadenados. El grupo IMSA adopta un estándar híbrido de desarrollo global y mul- 
tinacional debido al hecho de que es una corporación con áreas de negocios divisionales y con diversas alianzas, muchas de ellas en otros países. Ello hace que el posicionamiento estratégico corporativo reciba adaptaciones propias de acuerdo con las características anotadas. De forma específica la red alianza de tipo horizontal Enertec concilia las estrategias de las organizaciones socias para América Latina.

Ambiente.-Es un condicionante que actúa en conjunción con la estrategia que adopta un estándar global. En el caso del grupo CEMEX, esto significa que su ambiente de negocios es visto como un mercado único mundial, construyendo relaciones con proveedores, clientes y hasta competidores de tamaño y clase mundial. En FEMSA Logística, el ambiente se forma conjuntamente con clientes, proveedores y hasta competidores que actúan localmente dentro de México (nacionales o internacionales). Para el grupo IMSA, la relación con los socios depende de cada área de negocios y de sus respectivas subsidiarias que actúan dentro de México y en otros países, sea con inversiones propias directas, sea en sociedad, como es el caso de Enertec (global/multinacional).

Tecnología.-Este condicionante, esencialmente permite el engranaje de la red por medio de tecnología informática. En el caso del grupo CEMEX, emplea sus propios sistemas diseñados internamente, mientras FEMSA Logística desarrolla sus sistemas en sociedad con Oracle. El grupo IMSA, en razón de las múltiples redes de negocios que tiene, combina una base central con las áreas divisionales, las cuales se interconectan con sus respectivas subsidiarias. No obstante, debido a la complejidad de sus relaciones, sobre todo con socios diferentes en sus diversos negocios, se ve obligada a adquirir sistemas en cada caso, siendo estas consideraciones aplicadas por Enertec.

Cultura.- Este condicionante, en el contexto de las redes investigadas, varía mucho en función del estilo y de la idiosincrasia, en razón de tratarse de redes de negocios diferentes. El grupo CEMEX adopta una cultura general típicamente etnocéntrica, que se fundamenta en la «superioridad paternalista» de la sede matriz, que dirige cada subsidiaria según criterios centralizados y sin otorgar autonomía. El grupo FEMSA también adopta un criterio etnocéntrico en cada una de sus divisiones, las cuales aplican en su contexto de negocio la estandarización de cultura del grupo; de forma específica FEMSA Logística actúa preferentemente en el mercado de bebidas, y está adaptada a la cultura del mercado. En el grupo IMSA se percibe una situación cultural híbrida, etnocéntrica y policéntrica, ya que en cada área de negocios desarrolla actividades locales e internacionales con subsidiarias que tienen alianzas con otras socias que también pretenden compartir actividades con idiosincrasias diferentes, a través de una negociación constante. En el caso específico de Enertec se juntan culturas de los países de las socias de México, Estados Unidos, Alemania y de los países donde actúan sus subsidiarias, sean Brasil, Argentina, Venezuela, Colombia y Estados Unidos.

\subsubsection{Componentes}

Departamentalización.- Aunque visualmente los organigramas son funcionales, en la realidad las actividades corresponden a procesos de negocios. De forma es- 
pecífica, CEMEX estableció procesos de negocios en nueve áreas centrales administradas por células (e-groups) Comercialización, Contraloría fiscal, Concreto, Operaciones, Finanzas, Recursos Humanos, Contraloría operativa, Planeamiento, Abastecimiento. Estos procesos de negocios se aplican de manera estandarizada en las diversas subsidiarias. En el caso de FEMSA Logística, hay procesos centrales estandarizados: Desarrollo de Negocios, Implementación de Nuevos Negocios, Evaluación de Nuevos Negocios, bajo el contexto de procesos administrativos específicos de apoyo, pero en los procesos de consultoría que desarrolla específicamente con sus clientes hay flexibilidad y capacidad de adaptación. En el grupo IMSA todavía hay áreas funcionales, pero con tendencia a los procesos, sobre todo por las actividades de internacionalización y de alianzas, como es el caso de Enertec, la cual está en un proceso de evolución que tiende hacia una estructura por procesos.

Actividades de línea /asesoría.-El estándar de los tres grupos es de una plena integración. Así, el grupo CEMEX se integra a nivel global con cada una de sus subsidiarias, conciliando sus niveles corporativo, geográfico y de procesos. El grupo IMSA se establece principalmente en la matriz, asesorando cada una de las áreas de negocios en aspectos centrales como finanzas o jurídicos, y esas áreas también se unen con sus diversas subsidiarias individuales o en alianzas. Para FEMSA Logística, la integración de sus actividades de negocios es esencial y cada proyecto con socios implica una administración específica y conjunta con ellos.

Descripción de actividades.- El grupo CEMEX se caracteriza por desarrollar una estandarización intensa de sus procesos en sus operaciones mundiales. En el caso del grupo IMSA, hay una mezcla de estandarización en algunos aspectos centrales corporativos, como en las finanzas, pero en otros otorga flexibilidad a cada área de negocios. Así, Enertec recibe autonomía para desarrollar actividades tácticas y operacionales en conjunto con sus socios. En FEMSA Logística las actividades se establecen con flexibilidad de adaptación de los servicios a favor de socios-clientes específicos de cada proyecto de consultoría.

Cadena jerárquica.- Existe una tendencia hacia la horizontalización, lo que significa disminución de niveles y extinción de la «vieja pirámide». Así, el grupo CEMEX mezcla niveles funcionales con áreas geográficas y procesos, pero la autoridad es claramente definida y respetada. En el grupo FEMSA, la autoridad se autodefine en la base corporativa y en cada área divisional; específicamente en FEMSA Logística se basa en la integración de sus cuadros gerenciales y en la combinación entre la base central y sus zonas geográficas, dependiendo de cada proyecto. El grupo IMSA ejerce su autoridad en la sede central corporativa y otorga autonomía a cada área de negocios y a sus respectivas subsidiarias. En el caso específico de Enertec, la autoridad es conciliada entre las socias de la red/alianza, por consenso.

Nivel de centralización/descentralización.- En el grupo CEMEX se observa una elevada centralización en su sede corporativa matriz, sustentada en la estrategia global. En los grupos IMSA y FEMSA la centralización estratégica existe, pero táctica y operacionalmente otorgan autonomía a sus subsidiarias, como son 
los casos de Enertec y FEMSA Logística, respectivamente.

Amplitud administrativa.- Es difícil establecer un número específico de subordinados directos adscritos a una única jefatura, ya que cada configuración de la red obedece a criterios diferentes. En el caso del grupo CEMEX, los criterios son los funcionales, geográficos y de procesos. En el grupo IMSA los criterios son las áreas de negocios, el geográfico y la red/alianza en el caso concreto de Enertec. El grupo FEMSA también se basa en áreas de negocios, como es FEMSA Logística, dependiendo de las características del proyecto de consultoría con sus socios.

Comunicación.- Al respecto, se resalta el hecho de que la configuración en red de las diversas opciones de los grupos investigados sustenta sus actividades con el empleo de la tecnología informática. El grupo CEMEX desarrolló su sistema propio en versión 1.0 CEMEX Way. En FEMSA Logística también se estableció un sistema propio en sociedad con Oracle. En el grupo IMSA, en razón de su configuración diferenciada en áreas de negocios y diversas subsidiarias, se firman acuerdos de alianzas, pues se necesita diversificar y adquirir sistemas específicos para cada uno de ellos, como es el caso de la red/alianza Enertec. Se resalta también el hecho de que la comunicación personal es fundamental en cada una de las organizaciones investigadas, ya que ella complementa la comunicación integrada interna y externa con proveedores, clientes y hasta competidores locales y/o mundiales.

Capacidad decisoria.- Es producto de las características diseñadas y dinamizadas por cada grupo. En el grupo CEMEX se puede notar que las decisiones son rápidas en razón del proceso de estandarización y virtualización de las comunicaciones e informaciones. En FEMSA Logística las decisiones son ad hoc, flexibles al contexto del proyecto específico con cada socio-cliente. En IMSA las decisiones son hasta cierto punto conservadoras, porque el grupo tiene una actuación diferenciada en cada área de negocios y en sus relaciones de alianzas.

\section{Consideraciones finales}

A modo de epílogo, presentamos algunas consideraciones finales con la intención de establecer si se lograron los objetivos propuestos en el estudio.

Con relación al objetivo central, puede decirse que éste fue alcanzado a través del modelo teórico propuesto, que mostró una fuerte relación e integración entre las tres unidades temáticas. La propuesta presenta a la estructura organizacional en red como opción de nuevo diseño organizacional, teniendo como contexto de actuación a la alianza estratégica y donde de por sí suceden negociaciones permanentes entre las organizaciones socias. $\mathrm{Su}$ validez ha sido probada en los tres grupos corporativos analizados.

Con relación al primer objetivo específico, éste fue cumplido por medio de la construcción de conceptos y tecnologías ad hoc a este estudio, los cuales muestran claramente dicha interacción. En cuanto a la definición, se puede notar que existe una convergencia terminológica coherente de mutua influencia y simbiosis. Con relación a la tipología, fue adoptado un criterio estándar de relaciones horizontales $\mathrm{y}$ verticales. 
Con relación al segundo objetivo específico, en el desarrollo de cada condicionante se demuestra la enorme influencia a nivel macroorganizacional de la concepción propuesta en las tres unidades temáticas contempladas en el modelo.

Con relación al tercer objetivo específico, se demuestra a nivel microorganizacional que esta nueva propuesta adopta un perfil de diseño y dinámica nueva, renovada, sui generis, con relación a los estándares de diseño tradicionales.

De forma sucinta, podemos establecer algunas ideas básicas en cuanto a los asuntos esenciales encontrados en la investigación:

- Existe una estrecha correlación entre la estructura organizacional en red, las alianzas estratégicas y las negociaciones, evidenciada tanto en la teoría como en los casos desarrollados.
- Existe influencia de todos los condicionantes considerados en el modelo, en el diseño y dinámica de la estructura organizacional en red.

- Existe una evolución manifiesta de la estructura organizacional en red, evidenciada en el «nuevo» perfil de cada uno de los componentes del diseño y dinámica organizacional.

- Como se ha podido apreciar, el presente artículo ha cumplido satisfactoriamente con todas las consideraciones previamente establecidas, con lo cual pretende contribuir a una mejor comprensión de esta nueva propuesta de diseño organizacional en red, que si bien puede ser considerada como un tema under construcción, se va consolidando a un ritmo sostenido y creciente en el contexto de la administración. 


\section{Referencias bibliográficas}

ALBERTIN, A. L. 2000. Comercio eletrônico: modelos, aspectos e contribuições de sua aplicação. São Paulo: Atlas. 242 págs.

AMOROSO, R. 1994. Alianças e arcerias: uma abordagem baseada na aprendizagem organizacional. 128 págs. Dissertação (Mestrado em Administração), Programa de Pós-Graduação em Administração, Faculdade de Economia, Administração e Contabilidade, Universidade de São Paulo, São Paulo.

BARDIN, L. 1977. Análise de Conteúdo. Lisboa: Edições 70.227 págs.

BARNATT, C. 1996. Cyber Business: mindsets for a wired age. London: Wiley \& Sons. 244 págs.

BATEMAN, T. y SNELL, S. 1998. Administração: construindo vantagem competitiva. São Paulo: Atlas. 539 págs.

BOVET, D. y MARTHA, J. 2001. Redes de Valor. Tradução Márcia Tadeu. São Paulo: Negócio. 259 págs.

BRANDENBURGER, A. M. y NALEBUFF, B. J. 1996. Co-opetition. New York: Doubleday. 290 págs.

CÂNDIDO, G. A. 2001. Fatores críticos de sucesso no processo de formação, desenvolvimento e manutenção de redes interempresariais do tipo agrupamento industrial entre pequenas e médias empresas: um estudo comparativo de experiências brasileiras. 328 págs. Tese (Doutorado em Engenharia de Produção), Programa de Pós-graduação em Engenharia de Pro- dução, Universidade Federal de Santa Catarina, Florianópolis.

CASTELLS, M. 2000. A Sociedade em Rede. Tradução Roneide Venâncio Majer. 4a. ed. São Paulo: Paz e Terra. 617 págs.

CRESWELL, J. W. 1994. Research design: qualitative \& quantitative approaches. Sage. 227 págs.

DELlAGNELLO, E. L. y SILVA, C. M. de. 2000. Literatura sobre novas formas organizacionais: onde se encontram as evidencias empíricas de ruptura com o modelo burocrático de organizações. En: Encontro da Associação Nacional dos Programas de Pós-Graduação em Administração, Enanpad 24, 2000, Florianópolis, SC. Resumos. Florianópolis, SC: Anpad. 1 CDROM.

DEVLIN, G. y BLEACKY, M. 1998. Strategic alliances guidelines for success. Long Range Planning. London, October, vol. 21, n. ${ }^{\circ}$, págs. $18-23$.

DYER, J.; KALE, P. y SINGH, H. 2001. How to make strategic alliances work. MIT Sloan Management Review. Boston, Summer, págs. 37-43.

FREIRE, A. 1998. Estratégia. Lisboa: Verbo. 244 págs.

GERSTEIN, M. 1994. Das burocracias mecânicas às organizações em rede: uma viagem arquitetônica. En: Nadler, D. et. al. (coord.). Arquitetura Organizacional: a chave para a mudança empresarial. Tradução Waltensir Dutra. Rio de Janeiro: Campus. 265 págs. 
GHOSHAL, S. y BARTLETT, Ch. 2000. A organização Individualizada. Tradução Afonso Celso da Cunha Serra. Rio de Janeiro: Campus. 334 págs.

GIL, A. 1988. Como elaborar projetos de pesquisa. São Paulo: Atlas. 159 págs.

GNYAWALI, D. y MADHAVAN, R. 2001. Cooperative networks and competitive dynamics: a structural embeddennes perspective. Academy of Management Review. Vol. 26, n. ${ }^{\circ}$ 3, págs. 431-445.

GROSSE, R. y KUJAWA, D. 1992. International business: theory and managerial applications. $2^{\text {nd }}$ ed. Homewood (Estados Unidos): Irwin.

KATAOKA, H. de C. 1995. The effectiveness of interorganizational relationships in a network organization. 240 págs. Tesis (Doctorado en Administración), Facultad de Administración, University of Toronto, Canadá.

KEEGAN, W. y GREEN, M. 1999. Principios de marketing global. Tradução Sara Schwartz e Cecilia Bartalotto. São Paulo: Saraiva.

LEWIS, J. 1992. Alianças estratégicas: estruturando e administrando parcerias para o aumento da lucratividade. São Paulo: Pioneira. 359 págs.

LIPNACK, J. y STAMPS, J. 1992. Network: redes de conexões: pessoas conectandose com pessoas. Tradução Merle Scoss. São Paulo: Aquariana.

LOUFFAT OLIVARES, J. E. 1999. Uma contribuição ao estudo da interação da estrutura organizacional com a estrutura de carreiras nas organizações: o caso do Insti- tuto de Pesquisas Energéticas e Nucleares, IPEN. 199 págs más anexos. Dissertação (Mestrado em Administração), Programa de Pós-Graduação em Administração, Faculdade de Economia, Administração e Contabilidade, Universidade de São Paulo, São Paulo.

LUDKE, M. y ANDRE, M. 1988. Pesquisa em educação: abordagens qualitativas. 2a ed. São Paulo: Pedagógica Universitária. 99 págs.

McGUILL, M. y SLOCUM, Jr., J. 1995. A empresa mais inteligente: como construir uma empresa que aprende e se adapta ás necessidades do mercado. Tradução Bazan Tecnologia e Lingüística. Rio de Janeiro: Campus. 283 págs.

MARTINELLI, D. P. y ALMEIDA, A. P. 1997. Negociação: como transformar confronto em cooperação. São Paulo: Atlas. 219 págs.

MILES, R. y SNOW, Ch. 1992. Cause of failure in network organizations. California Management Review. Summer, págs. 53-72.

NADLER, David et al. 1994. Arqueitetura organizacional: a chave para a mudanca empresarial. Rio de Janiero: Campus. 256 págs.

NADLER, D. y TUSHMAN, M. A. 2000. Organização do futuro: As lições importantes do século $\mathrm{XX}$ e os próximos desafios que levarão ao novo desenho da empresa. HSM Management. São Paulo, janeiro-fevereiro, n. 18, págs. 58-67.

NOHRIA, N. y ECCLES, R. (eds.). 1992. Networks and organizations: structure, form and action. Boston: Harvard Business School. 544 págs. 
OLIVEIRA, M. (coord./OBI Consultora). 1994. Negociação: novos e velhos malabarismos. São Paulo: Nobel. 190 págs.

OVERHOLT, M. H. 2000. Flexibilidade e Vantagem Competitiva: Os arquétipos de novo desenho organizacional flexível e a geração de executivos que surgem com ele. HSM Management. São Paulo, janeiro-fevereiro, año 3 , n. ${ }^{\circ} 18$, págs. 68-72.

PECI, A. 1998. Emergência e proliferação de redes organizacionais: marcando mudanças no mundo de negócios. En: Encontro da Associação Nacional dos Programas de Pós-Graduação em Administração, Enanpad 22, 1998, Foz de Iguaçu, PR. Resumos. Foz de Iguaçu, PR: Anpad. 1 CD-ROM.

PINTO, E. P. 1994. Negociacao orientada para resultados: a conquista do entendimiento atraves de criterios legitmos e objetivos. 2a. ed. Sao Paulo: Atlas. 136 págs.

ROSS, J. y LORANGE, P. 1996. Aliancas estrategicas: formacao, implemmentacao e evolucao. Sao Paulo: Atlas. 258 págs.

SAVAGE, Ch. 1996. 5 geração de gerência: criando por meio do empresariamento virtual, do dinamismo das equipes e do conhecimento colocado em rede. Tradução Nelson Leocadio. São Paulo: Pioneira.
SEGIL, L. 1998. Strategic alliances for the 21 st. century. Strategic \& Leadership. September/October, págs. 12-37.

SELLTIZ, C. 1964. Métodos de pesquisa nas relações sociais. $2^{\mathrm{a}}$ ed. São Paulo: Pedagógica Universitária. 687 págs.

SILVA, M. A. da. 1994. Alianças e redes organizacionais: bases para construção, formas, interdependências e modelos organizacionais inerentes. 197 págs. Dissertação (Mestrado em Administração), Pontifícia Universidade Católica de São Paulo, São Paulo.

SPARKS, D. B. 1992. A dinâmica da negociação efetiva: como ser bem sucedido através de uma abordagem ganha-ganha. Tradução Reinaldo Guarany. São Paulo.

URY, W. 1999. Negociar na era da informação. HSM Management. São Paulo, julho-agosto, año 3, n. $^{\circ} 15$.

WILDEMAN, L. 1999. Organização virtual. HSM Management. São Paulo, julhoagosto, año 3 , n. ${ }^{\circ} 15$, págs. $74-80$.

YIN, Robert. 1989. Case study research: design and methods. California: Sage. 165 págs. 